\title{
Erratum to:
}

\section{Particle density and transition temperature of weakly interacting quantum gases}

\section{Eur Phys. J. B 88, 258 (2015), DOI: 10.1140/epjb/e2015-60365-1}

Renu Bala ${ }^{1}$, Sunita Srivastava ${ }^{2}$, and Kare Narain Pathak ${ }^{2, a}$

1 Department of Physics, DAV University, 144012 Jalandhar, India

${ }^{2}$ Centre for Advanced Study in Physics, Panjab University, 160014 Chandigarh, India

Received 2 June 2016

Published online 4 July 2016 - (C) EDP Sciences, Società Italiana di Fisica, Springer-Verlag 2016

Above equation (A.5) the paragraph should be read as

"It is just observed that if we use the change in chemical potential at the transition temperature using equations (A.2) and (A.4) in (A.1) for $\Delta z$, and use $z_{0}=1-x$ and take the limit $x \rightarrow 0$, we get the density strictly in the first order in scattering length as:"

Equation (A.5) remains same and there is no change at all in our conclusion.

\footnotetext{
${ }^{\text {a }}$ e-mail: pathak@pu.ac.in
} 